\title{
EFEITO DAS CONDIÇÕES DE ATMOSFERA CONTROLADA SOBRE A OCORRẾNCIA DE DEGENERESCÊNCIA EM MAÇÃ 'FUJI'
}

\author{
A. BRACKMANN'; A.A. SAQUET ${ }^{2,3}$ \\ 'Departamento de Fitotecnia-UFSM., CEP: 97119-900, Santa Maria, RS. \\ ${ }^{2}$ Graduando do Curso de Agronomia da UFSM
}

\begin{abstract}
RESUMO: $O$ trabalho fol conduzido durante o ano de 1993, com objetivo avaliar o efeito das temperaturas e concentraçōes de oxigênlo nas câmaras de atmosfera controlada (AC), sobre a manifestação da degenerescência e conservação da qualidade dos frutos da maçã 'Fuji', durante o armazenamento. Os frutos utilizados já haviam sido armazenados em câmaras de atmosfera normal (AN) durante 72 dias, quando entāo já apresentavam $17 \%$ de degenerescência interna. Foram entāo armazenados em duas temperaturas $\left(1^{\circ} \mathrm{C}\right.$ e $2{ }^{\circ} \mathrm{C}$ ), UR de $97 \%$ e diferentes condições de $\mathrm{AC}: 0 \% \mathrm{CO}$ ' $1 \% \mathrm{O}_{2}, 0 \% \mathrm{CO}_{2} / 1,5 \% \mathrm{O}_{2}$ e $0 \% \mathrm{CO}_{2} / 2 \% \mathrm{O}_{2}$. Após 6 meses de armazenamento, a temperatura de $1{ }^{\circ} \mathrm{C}$ e concentrações de oxigênio mais baixas $\left(0 \% \mathrm{CO}_{2} / 1 \% \mathrm{O}_{2}\right.$ e $\left.\% \mathrm{CO}_{2} / 1,5 \% \mathrm{O}_{2}\right)$ proporcionaram os menores percentuais de degenerescência interna e externa, mantendo a firmeza da polpa mais elevada. No entanto, a acidez titulável manteve-se mais elevada na temperatura de $2^{\circ} \mathrm{C}$. Os teores de sólid os solúveis totais foram mais elevados na temperatura de $2^{\circ} \mathrm{C}$ na presença de 1,5 e $2,0 \%$ de $\mathrm{O}_{2}$.
\end{abstract}

Descritores: maça 'Fuji', atmosfera controlada, degenerescência

\section{EFFECT OF CONTROLLED ATMOSPHERE CONDITIONS ON THE BREAKDOWN OF 'FUJI' APPLES}

\begin{abstract}
The main aim of this research was to evaluate the effect of temperatures and oxygen concentrations in controlled atmosphere (CA) storage on the quality, conservation and breakdown of 'Fuji' apples, during storage. Fruits used had already been stored in cold storage during $\mathbf{7 2}$ days, and exhihited $17 \%$ of internal breakdown. Afterwards they were stored at two different temperatures $\left(1{ }^{\circ} \mathrm{C}\right.$ and $\left.2{ }^{\circ} \mathrm{C}\right)$ and in different $\mathrm{CA}$ conditions: $0 \% \mathrm{CO}_{2} / 1 \% \mathrm{O}_{2}, 0 \% \mathrm{CO}_{2}$ $1.5 \% \mathrm{O}_{2}$ and $0 \% \mathrm{CO}_{2} / 2 \% \mathrm{O}_{2}$. After a six months storage, fruits at $1{ }^{\circ} \mathrm{C}$ and at lower oxygen concentrations $\left(0 \% \mathrm{CO}_{2} /\right.$ $1 \% \mathrm{O}_{2}$ and $0 \% \mathrm{CO}_{2} 1.5 \% \mathrm{O}_{2}$ ) showed less internal and external breakdown and had higher firmness than fruits that had been stored at $2{ }^{\circ} \mathrm{C}$ or at higher orygen concentrations $\left(0 \% \mathrm{CO}_{2} / 2 \% \mathrm{O}_{2}\right)$. Tritatable acidity, however, was higher at $2{ }^{\circ} \mathrm{C}$. Soluble solids were higher at $2^{\circ} \mathrm{C}$ with 1.5 and $2.0 \%$ of oxygen.
\end{abstract}

Key Words: apple 'Fuj]', controlled atmosphere, breakdown

\section{INTRODUÇĀO}

A cultivar Fuji representa cerca de $40 \%$ do total da maçã produzida no Brasil. Seus frutos apresentam epiderme vermelha, boa suculência, polpa firme e maturação tardia. Por sua alta qualidade, é muito aceita no mercado interno e no exterior.

Esta cultivar apresenta baixos niveis de respiração e produção de etileno, quando comparada com outras cultivares (BRACKMANN, 1993), tem boa conservação em câmaras frias de atmosfera normal (AN), porém, a qualidade é superior quando é armazenada $\mathrm{em}$ condições de atmosfera controlada (AC), onde mantém a firmeza da polpa e acidez mais elevadas. No entanto, $O$ armazenamento em $A C$ pode manifestar a degenerescência interna, distúrbio fisiológico que pode comprometer a qualidade, podendo depreciar até $50 \%$ da maçã armazenada.
Este distúrbio caracteriza-se por regiões internas da polpa do fruto com predominância de coloração castanho-escura, sintoma este que somente poderá ser constatado mediante o corte e exposição interna do fruto. Em muitos casos, poderá ocorrer a formação de pequenas cavidades na polpa do fruto, decorrente da morte de células por desidratação. Porém, quando o problema se torna mais grave, poderão ser percebidas manchas necróticas até na superficie externa dos frutos.

A degenerescência interna é um distúrbio fisiológico, com causas praticamente desconhecidas. Os fatores determinantes da degenerescência na maçã 'Fuji', estão relacionados com as condiçžes de campo durante o período de frutificação e com as condições de armazenamento.

A manifestação da degenerescência em maçã pode estar relacionada com a colheita muito tardia

${ }^{3}$ Bolsista do CNPq

Sci. agric., Piracicaba, 52(2):263-267, mai./ago. 1995 
dos frutos (SCHUHMACHER, 1975; STREIF, 1985), com a alta incidência de "Pingo de Mel" (DEWEY, 1962; FORTES \& PETRI, 1982; STAINER, 1990) e de "Bitter Pit" (ATKINSON, 1971). Frutos grandes também são mais susceptiveis ao distúrbio (DEWEY, 1962; STAINER, 1990). Também foi observado que altos niveis de nitrogênio e baixos niveis de cálcio e fósforo nos frutos aumenta a sua susceptibilidade (EBERT, 1984).

Em condições de câmara, a degenerescência em maçãs pode ser causada por altas concentraç̋̃es de $\mathrm{CO}_{2}$ (FIDLER, 1973). Este distúrbio em 'Fuji', também pode estar associado com dano por baixa temperatura no armazenamento (FIDLER, 1973; BENDER, 1990). No entanto, EBERT (1984) relata que o problema é um processo natural de senescência, que pode ser acelerado pela temperatura elevada das câmaras ou pelo resfriameno demorado dos frutos. CURRY (1991) avaliando diferentes concentrações de oxigênio $\left(1 \% \mathrm{O}_{2}, 1,5 \% \mathrm{O}_{2}\right.$ e $\left.2 \% \mathrm{O}_{2}\right)$ em 'Fuji', na temperatura de $1^{\circ} \mathrm{C}$, não verificou a ocorrência de degenerescência interna da polpa em nenhuma das concentrações de oxigênio.

As empresas produtoras de maçãs, normalmente realizam um acompanhamento da ocorrência de degenerescência, através da coleta e avaliação semanal ou quinzenal de amostras de frutos. Em anos de grande incidência do distúrbio, surgem dúvidas para os técnicos responsáveis pelo armazenamento com relação às providências que devem ser tomadas, quanto à temperätura e concentrações de oxigênio das câmaras, com o objetivo de amenizar a evolução do problema.

Em vista disso, o objetivo do presente trabatho é avaliar o efeito das temperaturas e concentrações de oxigênio nas câmaras de $\mathrm{AC}$, sobre a manifestação da degenerescência e conservação da qualidade dos frutos da maçã 'Fuji', durante o armazenamento.

\section{MATERIAL E METODOS}

$O$ experimento foi conduzido durante o período de julho a dezembro de 1993, no Núcleo de Pesquisa em Pos-Colheita (NPP) da Universidade Federal de Santa Maria (UFSM). Foram utilizados frutos procedentes de pomar comercial da empresa produtora de maçãs Pomifrai, localizada em Fraiburgo-SC. Os frutos já haviam sido armazenados em câmaras frias de atmosfera normal (frio + ar normal) durante um período de 72 dias, quando foi constatado a ocorrência média de $17 \%$ de frutos com sintomas de degenerescência interna. Os frutos foram então transportados em veículo tipo camioneta, acondicionados em caixas plásticas de $20 \mathrm{~kg}$ cada e armazenados em câmaras experimentais, com volume de 240 litros, pertencentes ao NPP, sob diferentes condições de $\mathrm{AC}$ e temperatura.

$O$ delineamento experimental utilizado foi inteiramente casualizado, com duas repetiçôes por tratamento. A unidade experimental foi composta de 60 frutos por tratamento.

Os tratamentos avaliados foram obtidos da combinação de duas temperaturas e três concentrações de oxigênio, conforme pode ser visualizado na TABELA 1.

As temperaturas de armazenamento das câmaras tiveram uma oscilação de $\pm 0,5^{\circ} \mathrm{C}$ em decorrência da instabilidade normal de funcionamento.

A umidade relativa durante $o$ armazenamento permaneceu em torno de $97 \%$. A concentração de $\mathrm{CO}_{2}$ foi mantida baixa $(<0,5 \%)$, devido a alta sensibilidade da cv. Fuji a altos níveis de $\mathrm{CO}_{2}$, que causam aumento da incidência de degenerescência.

As condiçðes de AC das câmaras foram estabelecidas através da realização do "pulldown", que consiste na retirada do oxigênio das câmaras através da injeção de nitrogênio, até a obtenção das concentraçðes pré-estabelecidas. Os niveis de gás

TABELA 1. Condições de armazenamento em atmosfera controlada sob as quais foram submetidos os frutos da maça 'Fuji'.

\begin{tabular}{cccc}
\hline Tratamento & Temperatura $\left({ }^{\circ} \mathrm{C}\right)$ & $\% \mathrm{CO}_{2}$ & $\% \mathrm{O}_{2}$ \\
\hline 1 & 1 & 0 & 1 \\
2 & 1 & 0 & 1,5 \\
3 & 1 & 0 & 2 \\
4 & 2 & 0 & 1 \\
5 & 2 & 0 & 1,5 \\
6 & 2 & 0 & 2 \\
\hline \hline
\end{tabular}


carbônico próximos a zero foram obtidos mediante a utilização de cal hidratada no interior da câmara de $\mathrm{AC}$, sendo este, responsável pela fixação do $\mathrm{CO}_{2}$ liberado pelo processo respiratório dos frutos. Já, para compensar o oxigênio consumido pela respiração, foi necessário injetar ar nas câmaras. Para a manutenção dos níveis destes gases durante o armazenamento, foi necessário a realização diária da análise e correção das concentrações. Para tal, foram utilizados analisadores de $\mathrm{CO}_{2}$ e $\mathrm{O}_{2}$, pelos quais foi circulado o gás de cada câmara que, após a análise, retornou a respectiva câmara.

Foi realizada uma análise de frutos no início do experimento (julho de 93), quando foi avaliado a incidência de degenerescência interna $\mathrm{e}$ a firmeza da polpa dos frutos. Após seis meses de armazenamento em $\mathrm{AC}$ (dezembro de 93), no final do experimento, foi avaliada a ocorrência de degenerescência interna, degenerescência externa e degenerescência total da polpa, firmeza da polpa, teor de sólidos solúveis totais e acidez titulável.

Para a determinação da firmeza da polpa, foi utilizado o penetrômetro motorizado com ponteira de $11 \mathrm{~mm}$, sendo realizadas duas leituras em cada fruto na região equatorial, após a retirada da epiderme.

Os teores de sólidos solúveis totais foram determinados com o auxílio do refratômetro manual, sendo a leitura realizada em graus brix.

A determinação da acidez titulável foi realizada mediante a titulação de $10 \mathrm{ml}$ de suco, diluídos em $100 \mathrm{ml}$ de água destilada, com uma solução de hidróxido de sódio $0,1 \mathrm{~N}$ até $\mathrm{pH} 8,1$.

Para a avaliação da ocorrência de degenerescência interna foram realizados vários cortes na secção transversal dos frutos, visando expor totalmente o interior da polpa para a avaliação. A degenerescência interna foi determinada através da contagem de frutos com qualquer tipo de sintoma na polpa do fruto, como cavidades ou manchas escurecidas.

A avaliação da ocorrência da degenerescência total e externa, foi realizada através da contagem dos frutos com sintomas visíveis. Foi considerado degenerescência total, o fruto que apresentava a epiderme e a polpa totalmente escurecidas, enquanto que, na degenerescência externa foram considerados os frutos com escurecimento da epiderme e a parte subjacente.

\section{RESULTADOS E DISCUSSÃO}

Os frutos armazenados a temperatura de $1^{\circ} \mathrm{C}$, apresentaram percentuais de degenerescência interna inferiores aqueles armazenados a $2^{\circ} \mathrm{C}$ (TABELA 2), estando de acordo com EBERT (1984), que afirma existir uma associação entre temperaturas mais elevadas de armazenamento com a incidência de degenerescência em maçãs. Porém, são contrários aos resultados de FIDLER (1973) e BENDER (1990), onde afirmam que a ocorrência deste distúrbio está associada à baixas temperaturas $\left(1^{\circ} \mathrm{C}\right) \mathrm{de}$ armazenamento. Portanto, o aumento da temperatura das câmaras de $\mathrm{AC}$, para prevenir o aumento da manifestação da degenerescência, como vem sendo recomendado por técnicos da área de pós-colheita, não é uma técnica que minimiza o problema, podendo inclusive, aumentá-lo.

Com relação aos teores de oxigênio das câmaras, verifica-se uma correlação entre o aumento da concentração deste gás e a ocorrência do distúrbio (TABELA 2). No entanto, os dados obtidos discordam daqueles encontrados por CURRY (1991), onde não foi verificado a ocorrência de degenerescência interna, mesmo quando as concentrações de $\mathrm{O}_{2}$ utilizadas atingiram niveis mais elevados $\left(2 \% \mathrm{O}_{2}\right)$. Provavelmente, isto tenha ocorrido em virtude dos frutos serem menos susceptiveis ao dano, ao contrário dos frutos que foram utilizados no presente trabalho, que já apresentavam sintomas internos, quando da instalação do experimento. A manifestação destes sintomas aumentou, possivelmente, pela aceleração da taxa respiratória decorrente dos teores de $\mathrm{O}_{2}$ mais elevados.

A manifestaçăo da degenerescência externa (TABELA 2), foi influenciada principalmente pela temperatura. $\mathrm{Na}$ temperatura de $1^{\circ} \mathrm{C}$ sua ocorrência foi inferior que a $2^{\circ} \mathrm{C}$. Baixo nível de $\mathrm{O}_{2}(1 \%)$, também reduziu sua manifestação na temperatura de $1^{\circ} \mathrm{C}$. É provável que a degenerescência externa, seja uma manifestação da senescência do fruto, já sugerido por EBERT (1984). A degenerescência total do fruto, foi na maioria dos casos, uma associação das duas formas de degenerescência, sendo provavelmente, consequência da elevação da temperatura e concentrações de oxigênio.

Os percentuais mais elevados de frutos sadios, foram obtidos mediante a utilização da temperatura de $1^{\circ} \mathrm{C}$ e concentrações de $1,5 \% \mathrm{O}_{2}$ (TABELA 
2). Isto significa que, a baixa temperatura e baixos niveis de oxigênio no armazenamento inibiram o avanço da degenerescêcia das maçãs já afetadas no início do experimento.

Mesmo não diferindo estatisticamente, a temperatura de $1^{\circ} \mathrm{C}$ proporcionou valores médios de firmeza da polpa superiores a temperatura de $2^{\circ} \mathrm{C}$ (TABELA 3). Também não houve diferença estatistica entre as três concentrações de $\mathrm{O}_{2}$, porém, houve uma tendência de 1 e $1,5 \% \mathrm{O}_{2} \mathrm{em}$ manter a firmeza da polpa em ambas as temperaturas mais elevada. LAU (1985), também verificou que maçãs armazenadas em temperatura baixa $(0$ a $\left.0,5^{\circ} \mathrm{C}\right)$ e níveis de oxigênio baixos $\left(1 \% \mathrm{O}_{2}\right.$ a $\left.1,5 \% \mathrm{O}_{2}\right)$, mantiveram a firmeza de polpa mais elevada, quando comparados aos frutos submetidos a $2,5 \%$ de $\mathrm{O}_{2}$.

A acidez titulável manteve-se mais elevada quando em temperatura de $2^{\circ} \mathrm{C}$.

Quanto aos teores de SST, praticamente não foi possivel verificar nenhuma influênicia dos tratamentos sobre este parâmetro (TABELA 3). Frutos armazenados a $2^{\circ} \mathrm{C}$ nas concentrações de $1,5 \mathrm{e}$ $2,0 \% \mathrm{O}_{2}$ tiveram níveis mais elevados de SST, provavelmente devido a maturação mais avançada destes frutos, pois temperaturas e concentrações de $\mathrm{O}_{2}$ mais elevadas estimulam a maturação.

TABELA 2. Efeito das temperaturas e concentrações de oxigênio das câmaras de AC, sobre a incidência de degenerescência em frutos da maçã cv. Fuji, durante o armazenamento.

\begin{tabular}{|c|c|c|c|c|c|c|c|}
\hline & $\begin{array}{r}\text { Trata } \\
\text { nentos }\end{array}$ & $\begin{array}{l}\text { Tempe- } \\
\text { ratura } \\
\left({ }^{\circ} \mathrm{C}\right)\end{array}$ & $\% \mathrm{CO}_{2} / \% \mathrm{O}_{2}$ & $\begin{array}{l}\text { \%Frutos } \\
\text { degener. } \\
\text { interna }\end{array}$ & $\begin{array}{l}\text { \%Frutos } \\
\text { degener. } \\
\text { externa }\end{array}$ & $\begin{array}{c}\text { \%Frutos } \\
\text { degener. } \\
\text { total }\end{array}$ & $\begin{array}{c}\text { \%Frutos } \\
\text { sadios }\end{array}$ \\
\hline \multirow[t]{7}{*}{ Início } & do & naz. em & 17,0 & & & & \\
\hline & 1 & 1 & $0 / 1$ & $2,8 \mathrm{~b}$ & $1,9 \mathrm{~d}$ & 38,3 & 57,0 a \\
\hline & 2 & 1 & $0 / 1,5$ & $10,6 \mathrm{a}$ & 4,9 cd & 18,8 & $65,6 \mathrm{a}$ \\
\hline & 3 & 1 & $0 / 2$ & $92 \mathrm{a}$ & 8,6 bc & 29,3 & $52,0 \mathrm{~b}$ \\
\hline & 4 & 2 & $0 / 1$ & $7,2 \mathrm{a}$ & $20,0 \mathrm{ab}$ & 25,4 & $47,3 \mathrm{bc}$ \\
\hline & 5 & 2 & $0 / 1,5$ & 8,2 a & $24,8 \mathrm{a}$ & 29,3 & $37,7 \mathrm{c}$ \\
\hline & 6 & $2^{2}$ & $0 / 2$ & $13,8 \mathrm{a}$ & $13,9 \mathrm{abc}$ & 25,9 & $46,4 \mathrm{bc}$ \\
\hline
\end{tabular}

As médias seguidas pelas mesmas letras na vertical, não diferem estatisticamente entre si pelo teste de Duncan ao nivel de $5 \%$ de probabilidade.

TABELA 3. Efeito das temperaturas e concentraç̄̃es de oxigênio das câmaras de AC, sobre as qualidades fisico-químicas de maçãs cv. Fuji.

\begin{tabular}{|c|c|c|c|c|c|}
\hline $\begin{array}{l}\text { Trata } \\
\text { mentos }\end{array}$ & $\begin{array}{l}\text { Tempe- } \\
\text { ratura } \\
\left({ }^{\circ} \mathrm{C}\right)\end{array}$ & $\% \mathrm{CO}_{2} / \% \mathrm{O}_{2}$ & $\begin{array}{l}\text { Firmeza polpa } \\
\text { (N) }\end{array}$ & SST\% & $\begin{array}{c}\text { Acidez } \\
\text { (meq/100ml) }\end{array}$ \\
\hline \multicolumn{3}{|c|}{ Inicio do armaz. em AC } & 59,7 & & \\
\hline 1 & 1 & $0 / 1$ & 53,4 a & $12,9 \quad b$ & $1,9 \mathrm{a}$ \\
\hline 2 & 1 & $0 / 1,5$ & $51,5 \mathrm{a}$ & $13,0 \mathrm{~b}$ & $1,9 \mathrm{a}$ \\
\hline 3 & 1 & $0 / 2$ & $51,2 \mathrm{a}$ & $13,1 \quad b$ & $1,8 \mathrm{a}$ \\
\hline 4 & 2 & $0 / 1$ & $49,3 \mathrm{a}$ & 12,9 b & $2,3 \mathrm{a}$ \\
\hline 5 & 2 & $0 / 1,5$ & $52,9 \mathrm{a}$ & 13,5 a & $2,3 \mathrm{a}$ \\
\hline 6 & 2 & $0 / 2$ & $48,6 \mathrm{a}$ & 13,2 a b & $2,0 \mathrm{a}$ \\
\hline
\end{tabular}

As médias seguidas pelas mesmas letras na vertical, não diferem estatisticamente entre si pelo teste de Duncan ao nivel de $5 \%$ de probabilidade. 


\section{CONCLUSÕES}

Através dos resultados obtidos no presente trabalho, é possivel concluir que em frutos já afetados pela degenerescência interna:

- O distúrbio aumenta com o aumento de oxigênio na câmara na faixa de 1,0 a $2,0 \%$.

- $\mathrm{O}$ aumento da temperatura de $1^{\circ} \mathrm{C}$ para $2^{\circ} \mathrm{C}$ intensifica a manifestação do problema.

- Quando a degenerescência for constatada em câmaras frigoríficas comerciais, sua evolução pode ser retardada através do abaixamento da temperatura para $1^{\circ} \mathrm{C}$ e concentrações de oxigênio de $1,5 \%$.

\section{REFERÊNCLAS BIBLIOGRÁFICAS}

ATKINSON, J.D. Diaseases of tree fruits in New Zealand. Wellington: Clark \& Matheson, 1971. 406p.

BENDER, R.J. What problems may arise from storing 'Fuji' apples in CA ? In: INTERNATIONAL HORTICULTURAL CONGRESSS-ABSTRACTS, 23 ., Firenze, 1990, Abstracts... Firenze, 1990. v.2, p.3328.

BRACKMANN, A. $\mathrm{CO}_{2}$, ethylene and aroma volatiles production by apple varieties. In: POSTHARVEST 93, INTERNATIONAL SYMPOSIUM. Kecskemét, Hungary, 1993. Abstracts... Alexandria: American Society for Horticultural Science, 1993. v.1, p.2.

CURRY, E.A. Effect of harvest date and oxygen level on storability of late season apple cultivars. In: INTERNATIONAL CONTROLLED ATMOSPHERE RESEARCH CONFERENCE, 5., Washington, 1991. Proceedings... v.1, p.103-109, 1991.
DEWEY, D.H. Factors affecting the quality of Jonathan apple stored in controlled atmosphere. In: INTERNATIONAL HORTICULTURAL CONGRESS, 16., Brussels, 1962, Proceedings... Belgium, 1962. v.1, p.278.

EBERT, A. Distúrbios fisiológicos. In: CURSO SOBRE A CULTURA DA MACIEIRA, 2., Caçador, 1994. Palestras... Florianópolis: EMPASC, 1984. p.129-134.

FIDLER, J.C. Conditions of storage. In: FIDLER, J.C., WILKINSON, B.G., EDNEY, K.L and SHARPLES, R.O. The biology of apple and pear storage, Wallingford: Commonwealth Agricultural Bureaux, 1973, p.1-51.

FORTES, G.R.L.; PETRI, J.L. Distúrbios fisiológicos em macieira e seu controle. Florianópolis: EMPASC/ ACARESC, 1982. 34p. (Boletim Técnico, 3).

LAU, O.L. Storage procedures, low oxygen and low carbon dioxide atmospheres on storage quality of 'Golden Delicious' and 'Delicious' apples. Journal of the American Society for Horticultural Science, Geneve, v.110, n.4, p.541-547, 1985.

SCHUHMACHER, R. Die Fruchtharkeit der Geholze. Ulm: Ulmer, 1975.197p.

STAINER, R. Gala, Braeburn und Fuji. Obstbau-Weinbau, Bonn, v.2, p.40-42, 1990.

STREIF, J. Qualitatsprobleme bei Apfellagerum. Obstbau, Bonn, v. 10, p.177-179, 1985.

Recebido para publicação em 01.06 .94

Aceito para publicação em 05.09 .94 